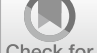

Check for

updates

Cite as

Nano-Micro Lett.

(2021) 13:146

Received: 10 March 2021

Accepted: 11 May 2021

Published online: 19 June 2021

(C) The Author(s) 2021

\section{Iron-Imprinted Single-Atomic Site Catalyst-Based Nanoprobe for Detection of Hydrogen Peroxide in Living Cells}

\author{
Zhaoyuan Lyu ${ }^{1}$, Shichao Ding ${ }^{1}$, Maoyu Wang ${ }^{2}$, Xiaoqing Pan ${ }^{3}$, Zhenxing Feng ${ }^{2}$, \\ Hangyu Tian ${ }^{1}$, Chengzhou Zhu ${ }^{1}$, Dan $\mathrm{Du}^{1}{ }^{凶}$, Yuehe $\operatorname{Lin}^{1} \bowtie$
}

\title{
HIGHLIGHTS
}

- A facile ion-imprinting method (IIM) is used to synthesize the isolated Fe-N-C single-atomic site catalyst (IIM-Fe-SASC), which mimics the natural enzyme-like active site and shows excellent peroxidase-like activity.

- The ion-imprinting process can precisely control ion at the atomic level and form numerous well-defined single-atomic Fe-N-C sites.

- The IIM-Fe-SASC has been successfully used as the nanoprobe for in situ $\mathrm{H}_{2} \mathrm{O}_{2}$ detection generated from MDA-MB-231 cells.

\begin{abstract}
Fe-based single-atomic site catalysts (SASCs), with the natural metalloproteases-like active site structure, have attracted widespread attention in biocatalysis and biosensing. Precisely, controlling the isolated single-atom Fe-N-C active site structure is crucial to improve the SASCs' performance. In this work, we use a facile ionimprinting method (IIM) to synthesize isolated Fe-N-C single-atomic site catalysts (IIM-Fe-SASC). With this method, the ion-imprinting process can precisely control ion at the atomic level and form numerous well-defined single-atomic Fe-N-C sites. The IIM-Fe-SASC shows better peroxidase-like activities than that of non-imprinted references. Due to its excellent properties, IIM-Fe-SASC is an ideal nanoprobe used in the colorimetric biosensing of hydrogen peroxide $\left(\mathrm{H}_{2} \mathrm{O}_{2}\right)$. Using IIM-Fe-SASC as the nanoprobe, in situ detection of $\mathrm{H}_{2} \mathrm{O}_{2}$ generated

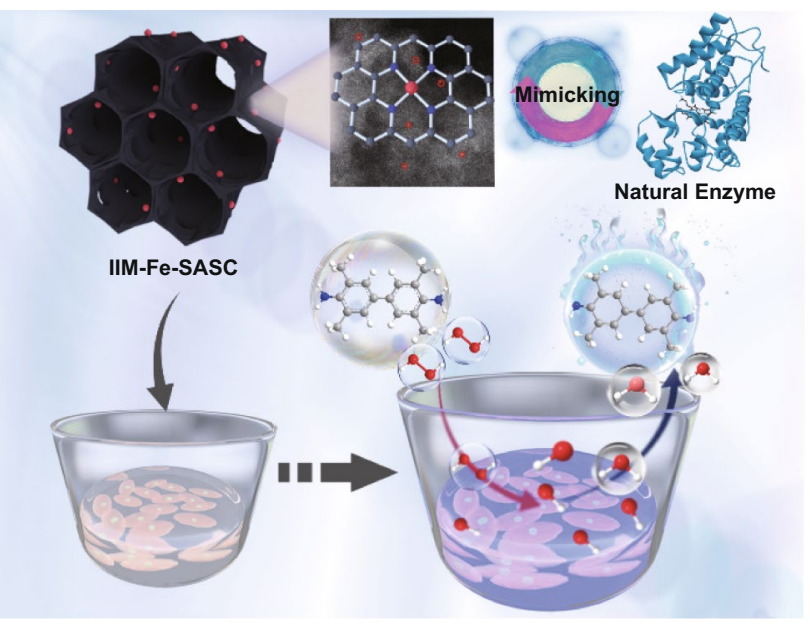
from MDA-MB-231 cells has been successfully demonstrated with satisfactory sensitivity and specificity. This work opens a novel and easy route in designing advanced SASC and provides a sensitive tool for intracellular $\mathrm{H}_{2} \mathrm{O}_{2}$ detection.
\end{abstract}

KEYWORDS Single-atomic site catalysts; Nanoprobe; Peroxidase-like activities; Biosensing; Living cell

Zhaoyuan Lyu and Shichao Ding have contributed equally to this work.

$\triangle$ Dan Du, annie.du@wsu.edu; Yuehe Lin, yuehe.lin@wsu.edu

1 School of Mechanical and Materials Engineering, Washington State University, Pullman, WA 99164, USA

2 School of Chemical, Biological, and Environmental Engineering, Oregon State University, Corvallis, OR 97331, USA

3 Irvine Materials Research Institute (IMRI), University of California, Irvine, CA 92697, USA 


\section{Introduction}

Hydrogen peroxide $\left(\mathrm{H}_{2} \mathrm{O}_{2}\right)$, playing an important role in physiological processes and as a messenger molecule for cellular effects, is crucial for immune response and cell growth/ senescence $[1,2]$ and also serve as potential biomarkers for diagnosis or monitoring diseases, such as diabetes, cancers, inflammation, cardiovascular and neurodegenerative diseases $[3,4]$. Therefore, sensing $\mathrm{H}_{2} \mathrm{O}_{2}$ generated from living cells with high sensitivity and specificity will be of vital clinical value for disease diagnosis and better comprehension of disease mechanisms [5, 6]. However, due to the relatively low concentration of $\mathrm{H}_{2} \mathrm{O}_{2}$ in physiological environments, in vivo $\mathrm{H}_{2} \mathrm{O}_{2}$ sensing for clinical applications remains a challenge [7]. Developing highly sensitive $\mathrm{H}_{2} \mathrm{O}_{2}$-responsive probes has paramount importance for biosensing in living cells.

Single-atomic site catalysts (SASCs) containing atomically dispersed metal active sites demonstrate distinctive advantages in catalytic activities and selectivity for various catalytic reactions [8-14]. Recently, SASCs with peroxidase-like activity characteristics have attracted numerous attentions in the biosensing field due to their attractive properties of high stability and unprecedented catalytic performance toward $\mathrm{H}_{2} \mathrm{O}_{2}$ [15-18]. Therefore, Fe-based SASCs are considered as substitutes of natural horseradish peroxidase (HRP) owing to their maximum specific activity and atomic utilization and have been applied in biosensing and bioremediation [19-22]. For example, we reported a Fe-based SASC linked immunosorbent assay for early detection of Alzheimer's disease, and an ultralow detection limit was achieved [23]. To rationally design Fe-based SASCs, researchers are usually devoted to selecting special precursors that either already contain single-atom metal species or use the coordination between the complex ligands and surface groups of support materials [24, 25]. Moreover, adsorbing iron ions to bulk materials or using a top-down synthetic method to peel off iron from metal bulk can also synthesize SASCs [26-28]. These methods have drawbacks of using expensive organic macromolecule complexes and running the risk of aggregating single-atom metal species into nanosized metal counterparts [29, 30]. The resulted SASCs either require high cost or possess a relatively low density of the active sites, which limit their large-scale practical applications. Therefore, new strategies for constructing coordination sites for preparing Fe-based SASCs are urgently needed.
Ion-imprinting technology (IIT) is a type of molecularly imprinted technology that involves self-assembly of the interested ion (the template), complementary functional monomers and cross-linkers to synthesize imprinted materials [31-33]. Generally, pre-polymerized complex systems can be formed during the preassembled system with each isolated template ion interacting with function monomer independently, and the template ions are embedded and isolated in the cross-linked matrix after the polymerization process. Due to the pro-coordination process between ions and functional monomers, the activate sites are precisely controlled at the atomic level and high-density single-atom irons are obtained. Hence, based on the advantages of IIT, it is believed that utilizing IIT can effectively confine the ion in the matrix and achieve a high yield of SASCs with a low-cost and straightforward process [34].

Hence, a facile ion-imprinting approach was used to synthesize the Fe-based single-atomic site catalyst (IIM-Fe-SASC) [35], and the developed Fe-SASC was used as a nanoprobe for in situ intracellular $\mathrm{H}_{2} \mathrm{O}_{2}$ detections (Fig. 1a). For synthesizing the IIM-Fe-SASC, the mesoporous silica was used as the matrix in the imprinted materials to prevent aggregation of the isolated iron ions. The [3-(2-Aminoethylamino)propyl]trimethoxysilane (A-Tri-EOS) was selected as functional monomers for that it could provide coordination sites to immobilize the iron atom. Precisely, controlled high-density single-atomic activate sites were achieved during the pro-coordination process between iron ions and A-Tri-EOS. IIM-Fe-SASC with inherent peroxidase-like activity could catalyze $\mathrm{H}_{2} \mathrm{O}_{2}$ to reactive oxygen species. In this paper, we successfully applied IIMFe-SASC as the sensing probe in a typical colorimetric assay to detect $\mathrm{H}_{2} \mathrm{O}_{2}$ with ultrahigh sensitivity and specificity. The IIM-Fe-SASC showed better peroxidase-like ability than that of non-imprinted references. Importantly, in situ detection of $\mathrm{H}_{2} \mathrm{O}_{2}$ generated from breast cancer cells (MDA-MB-231) was performed using the IIM-Fe-SASC-based assay, which demonstrates the practical clinic applications of SASC nanoprobe.

\section{Experimental}

\subsection{Preparation of Single-Atomic Site Catalyst Nanoprobe}

To prepare IIM-Fe-SASC, the mesoporous structuredirecting micelles (solution 1) was first prepared by adding $0.6 \mathrm{~g}$ cetyltrimethylammonium bromide $(\mathrm{CTAB})$ in a 

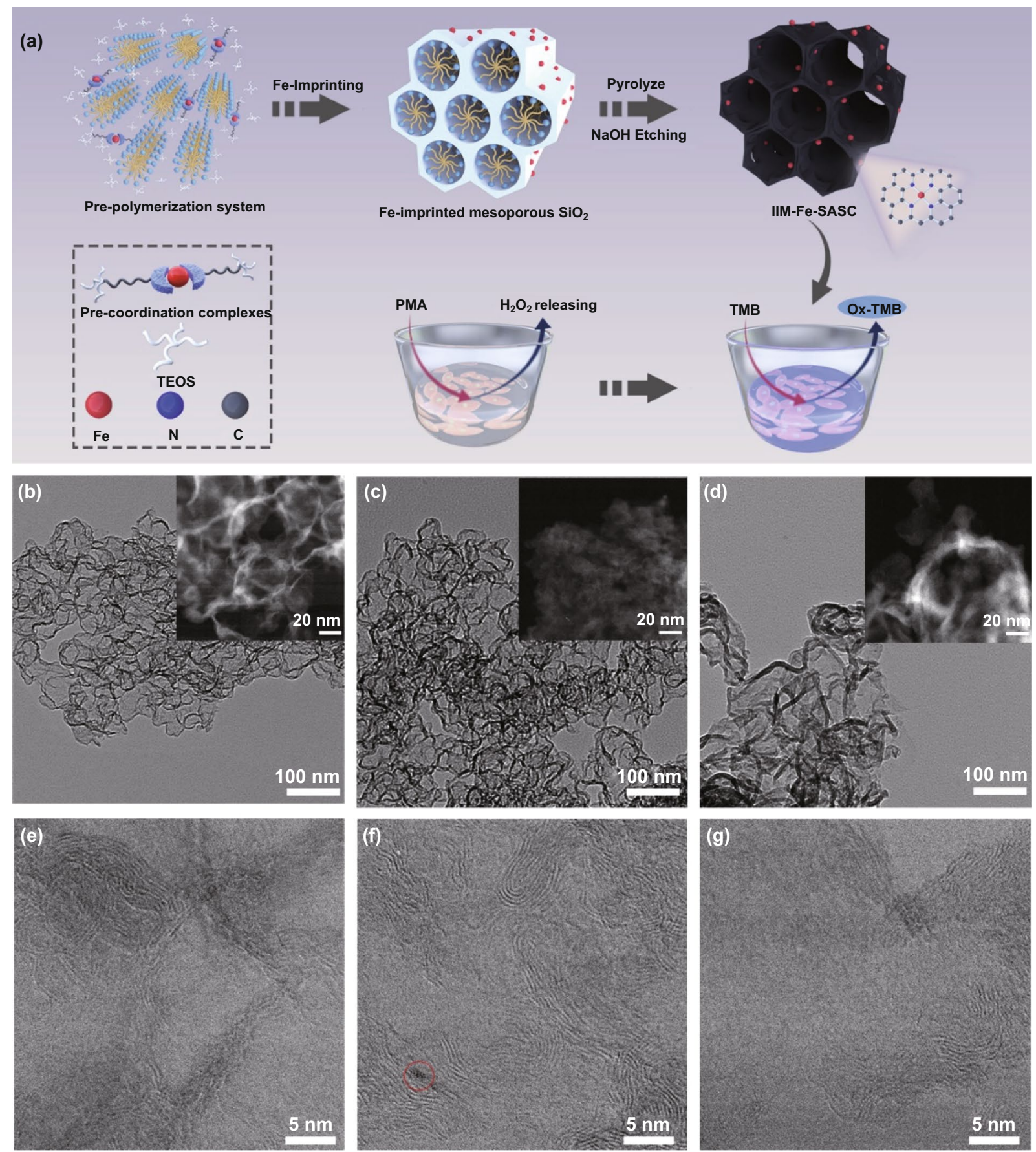

Fig. 1 a Schematic diagram of the process steps for IIM-Fe-SASC nanoprobe synthesis and in situ intracellular hydrogen peroxide detection. b-d TEM images of IIM-Fe-SASC, NIM-Fe-SASC, and NIM in different resolutions. e-g STEM bright-field images for IIM-Fe-SASC, NIMFe-SASC, and NIM at higher magnification

$\mathrm{NaOH}$ solution under vigorous stirring. $11 \mathrm{mg}$ ion template $\mathrm{Fe}\left(\mathrm{NO}_{3}\right)_{3}$ and $0.25 \mathrm{~mL}$ functional monomers A-Tri-EOS were dissolved together and shaken for $15 \mathrm{~min}$ (solution 2), aiming to form the pre-coordination complexes of iron ions-functional monomers. Then, solution 2 and
$2.5 \mathrm{~mL}$ cross-linking agent tetraethyl orthosilicate (TEOS) were added dropwise to solution 1, and the Fe-imprinted mesoporous $\mathrm{SiO}_{2}$ could be obtained through filtration after reaction for $2 \mathrm{~h}$. Meanwhile, to analyze and compare the performance of IIM-Fe-SASC, two more materials were 
synthesized and used as compare samples: Fe-based SASC synthesized using an adsorbing method (NIM-Fe-SASC) and a non-imprinted nanomaterial without $\mathrm{Fe}$ ions (NIM). For the adsorbing method, non-imprinted mesoporous $\mathrm{SiO}_{2}$ was prepared with the same route without adding iron ions. Subsequently, the obtained mesoporous $\mathrm{SiO}_{2}$ was used to absorb the same amount of $\mathrm{Fe}\left(\mathrm{NO}_{3}\right)_{3}$ solution and then the Fe-adsorbed mesoporous $\mathrm{SiO}_{2}$ was collected after filtration. The Fe-imprinted mesoporous $\mathrm{SiO}_{2}$, Fe-adsorbed mesoporous $\mathrm{SiO}_{2}$ and mesoporous $\mathrm{SiO}_{2}$ were pyrolyzed at $900{ }^{\circ} \mathrm{C}$ for $30 \mathrm{~min}$ in $\mathrm{N}_{2}$ atmosphere and then $30 \mathrm{~min}$ in $\mathrm{NH}_{3}$ atmosphere. Then, the isolation matrix $\mathrm{SiO}_{2}$ was removed by soaking in $3 \mathrm{M} \mathrm{NaOH}$ at $80{ }^{\circ} \mathrm{C}$ for $48 \mathrm{~h}$. The synthesized samples are named IIM-Fe-SASC (by imprinting method), NIM-Fe-SASC (by adsorbing method) and NIM (without adding Fe iron), respectively. Further, specific evaluating peroxidase-like activity processes are described in Supplementary Information.

\subsection{Detect $\mathrm{H}_{2} \mathrm{O}_{2}$ Produced from the Breast Cancer Cells}

In order to evaluate the $\mathrm{H}_{2} \mathrm{O}_{2}$ released from MDA-MB-231 cells, the cells were placed in a 96-well plate for $24 \mathrm{~h}$. After that, the plates were washed three times using PBS solution. Then, PMA solution $(20 \mu \mathrm{L}, 2 \mu \mathrm{M})$ and $100 \mu \mathrm{L}$ PBS were sequentially added and incubated for $30 \mathrm{~min}$. Finally, IIM-Fe-SASC $\left(10 \mathrm{mg} \mathrm{mL}^{-1}, 50 \mu \mathrm{L}\right)$, TMB $(1 \mathrm{mM}, 100 \mu \mathrm{L})$ and HAC-NaAC buffer (100 $\mu \mathrm{L}, \mathrm{pH} 4.0)$ were subsequently added and incubated for $5 \mathrm{~min}$. Finally, a multi-mode reader was used to record the absorbance at $652 \mathrm{~nm}$.

\subsection{Intracellular Imaging}

For live/dead cell imaging, MDA-MB-231 cells were seeded in 6-well plates and incubated overnight at $37^{\circ} \mathrm{C}$ in a cell culture incubator. Add IIM-Fe-SASC nanoprobe or TMB with $10 \mathrm{mg} \mathrm{mL}^{-1}$ and $1 \mathrm{mM}$ and incubate in the dark for $30 \mathrm{~min}$, respectively. Subsequently, the calcein-AM $(2 \mu \mathrm{M})$ and EthD-1 $(4 \mu \mathrm{M})$ solutions from the live/dead viability kit were added to each well and incubated for $15 \mathrm{~min}$. Finally, after washing thoroughly with PBS, observe the cells with CLSM (Leica TCS SP8). Here, the green fluorescence from Calcein-AM represents living cells, and the red fluorescence from EthD-1 represents dead cells. For the intracellular ROS imaging, DCFH-DA was used to stain cells as a ROS fluorescent probe. MDA-MB-231 cells were seeded in 12-well plates and incubated for $24 \mathrm{~h}$ in a cell culture incubator containing 5\% $\mathrm{CO}_{2}$ and $95 \%$ humidity. Then, the cells were incubated with IIM-Fe-SASC nanoprobe for $4 \mathrm{~h}$ under $0,2.5,5$ and $10 \mu \mathrm{g} \mathrm{mL}^{-1}$, respectively. Herein, the IIMFe-SASC nanoprobe was broken down to nanosize via an intense ultrasound treatment for use in endocytosis. Then, $10 \mu \mathrm{M}$ DCFH-DA was added to each well, followed by incubation for $20 \mathrm{~min}$. The cells were washed using PBS. Finally, fluorescence images were obtained by CLSM.

\section{Results and Discussion}

\subsection{Materials Characterizations}

Transmission electron microscopy (TEM) was used to study the structures and morphologies of as-prepared samples. Figure S1 shows well-defined mesoporous structures in both $\mathrm{Fe}$-imprinted and non-Fe-imprinted mesoporous $\mathrm{SiO}_{2}$ precursors. No obvious structural difference between them is found, indicating that the sol-gel polymerization and mesoporous $\mathrm{SiO}_{2}$ precursor structures are not affected when adding Fe ions. The obtained IIM-Fe-SASC, NIMFe-SASC and NIM show the inhomogeneous structure (Fig. 1b-d). Moreover, in Fig. 1e-g, distorted graphite layers were observed by scanning TEM (STEM), which makes the catalysts rich in defects and nanopores, thus accommodates a large amount of single-atom active sites. The STEM images of IIM-Fe-SASC are the same as that of NIM, where no nanoparticles are observed, suggesting that iron atoms embed into the IIM-Fe-SASC as dispersive isolated atoms. However, nanocrystal can be found in NIM-Fe-SASC and is marked in the red circle in Fig. 1f, illustrating that the adsorption method can easily produce metal clusters and are hard to remove. X-ray diffraction (XRD) pattern demonstrates that the IIM-Fe-SASC possesses nanoparticle-free features (Fig. S2).

To further prove the state of single iron atom, aberrationcorrected high-angle annular dark-field STEM (HAADFSTEM) was employed to investigate the wall structure of the as-made IIM-Fe-SASC and NIM-Fe-SASC at the atomic level. For IIM-Fe-SASC, as marked in red circles in Fig. 2a, uniformly dispersed single-atom Fe sites show on the carbon matrix. Nevertheless, NIM-Fe-SASC, prepared by the 
traditional adsorption method, has both single iron atoms and some stacked metal crystals (Fig. 2b), further demonstrating that the doped Fe species are not purely single atoms. Elemental composition and distribution in IIM-FeSASC were detected by auxiliary energy-dispersive X-ray spectroscopy (EDS) elemental analysis. Figure 2c shows the corresponding element maps of carbon, nitrogen and iron in IIM-Fe-SASC. All elements are uniformly distributed in the IIM-Fe-SASC, indicating that nitrogen coordinated with Fe atoms can be doped into the carbon matrix. The Fe atom loading is confirmed as $2.12 \mathrm{wt} \%$, which was measured by inductively coupled plasma mass spectrometry (ICP-MS).
X-ray absorption spectroscopy (XAS) measurements $[36,37]$ were performed to determine the local structural and electronic states of $\mathrm{Fe}$ atoms in the as-synthesized catalysts. The absorption edge of Fe K-edge X-ray absorption near edge structure (XANES) spectrum of IIM-Fe-SASC is located at higher energy compared with that of $\mathrm{Fe}$ foil, but in between two reference samples $\left(\mathrm{FeO}\right.$ and $\left.\mathrm{Fe}_{2} \mathrm{O}_{3}\right)$, indicating that the $\mathrm{Fe}$ atoms in IIM-Fe-SASC have a positive charge (Fig. 2d) that is between +2 and +3 . Note that, the absorption edge of IIM-Fe-SASC almost overlaps with that of FePc, which may indicate the similarity of their local structure surrounding $\mathrm{Fe}$ atoms. Moreover, in Fig. 2e, the
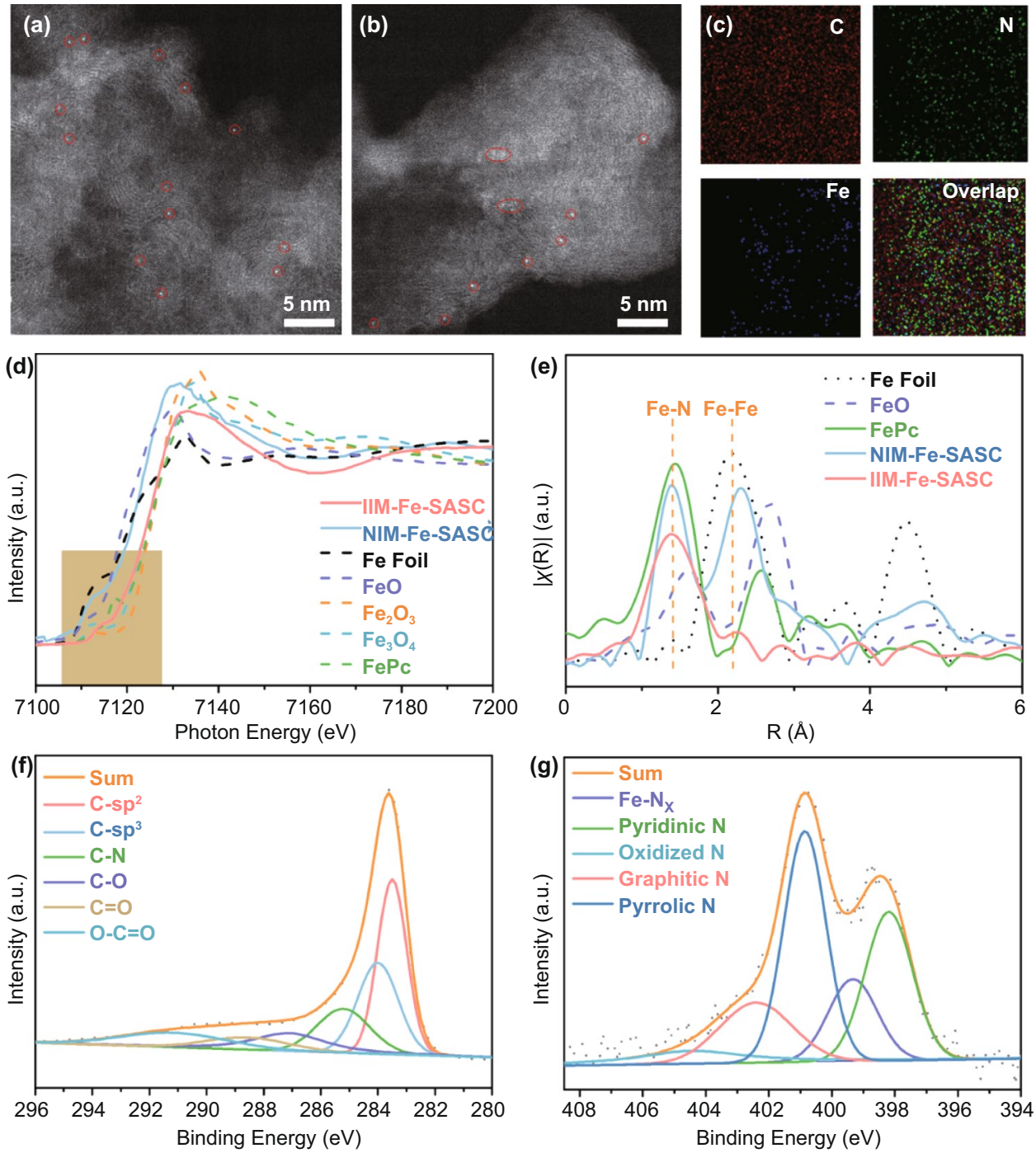

Fig. 2 a, b HAADF-STEM images of IIM-Fe-SASC and NIM-Fe-SASC, respectively. c Elemental -mapping images of IIM-Fe-SASC. d Fe K-edge XANES spectra of IIM-Fe-SASC, and reference samples of FePc, Fe foil, FeO, $\mathrm{Fe}_{2} \mathrm{O}_{3}$ and $\mathrm{Fe}_{3} \mathrm{O}_{4}$. e FT $k^{2}$-weighted EXAFS R-space spectra of IIM-Fe-SASC, NIM-Fe-SASC, FePc, Fe foil, and $\mathrm{Fe}_{2} \mathrm{O}_{3}$. f, g C $1 s$ and N $1 s$ XPS spectra of IIM-Fe-SASC 
Fourier-transformed (FT) $k^{2}$-weighted EXAFS curve of IIMFe-SASC only shows a main peak at about $1.4 \AA$, which is aligned with the $\mathrm{Fe}-\mathrm{N}$ peak in the $\mathrm{FePc}$ reference sample, suggesting that $\mathrm{Fe}$ is in single-atom dispersed form with $\mathrm{Fe}-\mathrm{N}$ bonding. In comparison, besides the $\mathrm{Fe}-\mathrm{N}$ scattering path, a Fe-Fe peak at $2.2 \AA$ (comparing with Fe foil) exists in NIM-Fe-SASC, demonstrating Fe exists as both $\mathrm{Fe}-\mathrm{N}_{x}$ motifs and metallic Fe clusters. These results are consistent with the HAADF-STEM results shown above. It is also noted that the $k$-space EXAFS oscillations (Fig. S3) reveal that IIM-Fe-SASC spectrum is different from those of Fe foil and $\mathrm{Fe}$ oxides, but similar to that of $\mathrm{Fe}$ single-atom reference FePc, which is mainly due to the fact that the selected functional monomers effectively coordinate iron atoms during the ion-imprinting process [24]. The chemical composition of the obtained IIM-Fe-SASC was conducted by X-ray photoelectron spectroscopy (XPS). The high-resolution C 1 s spectrum of the IIM-Fe-SASC (Fig. 2f) can be deconvoluted into four components of C-sp $p^{2}(283.6 \mathrm{eV}), \mathrm{C}-s p^{3}(284.0 \mathrm{eV})$, $\mathrm{C}-\mathrm{N}(285.2 \mathrm{eV}), \mathrm{C}-\mathrm{O}(287.1 \mathrm{eV}), \mathrm{C}=\mathrm{O}(288.6 \mathrm{eV})$ and $\mathrm{O}-\mathrm{C}=\mathrm{O}(289.1 \mathrm{eV})$ [38-40]. The ratio of C-sp $p^{2}$ in IIM-FeSASC is $33.4 \%$, much lower than the reported high graphitized carbon materials (like graphene) [41, 42], indicating that the IIM-Fe-SASC has a lower degree of graphitization and abundant defects and edges. Raman spectra were also used to study graphitizing degrees (Fig. S4). A strong D band and the relatively high-intensity ratio of $\mathrm{D}$ band to $\mathrm{G}$ band ( 0.91) further demonstrate the numerous existed defects and structural imperfections of IIM-Fe-SASC [43]. The complex N $1 s$ spectrum of IIM-Fe-SASC is deconvoluted into several main peaks (Fig. 2g), which correspond to pyridinic $\mathrm{N}(398.2 \mathrm{eV})$, pyrrolic $\mathrm{N}(400.9 \mathrm{eV})$, graphitic $\mathrm{N}$ (402.4 eV) and oxidized N (404.7 eV), respectively. [24, 44] Most important, a spectral valley between two dominating pyridinic peak and pyrrolic peak at $399.3 \mathrm{eV}$ indicates the presence of $\mathrm{Fe}-\mathrm{N}_{x}$ single-atom sites, [45] which is in good agreement with the result of EXAFS. Besides, the Fe $2 p$ spectra are shown in Fig. S5, which further illustrates the successful Fe doping.

\subsection{Peroxidase-like Activities Evaluation}

The peroxidase-like activities of the IIM-Fe-SASC, NIMFe-SASC and NIM are verified, and the results of the chromogenic reaction are shown in Fig. 3a. The obvious color change of IIM-Fe-SASC can be observed and is caused by the oxidation of colorless substrates to their corresponding oxidized products. Notably, NIM cannot trigger any chromogenic reaction regardless of the existence of $\mathrm{H}_{2} \mathrm{O}_{2}$, which proves that the peroxidase-like property of IIM-Fe-SASC is mainly originated from $\mathrm{Fe}-\mathrm{N}_{x}$ sites. The peroxidaselike activity of IIM-Fe-SASC and control samples were conducted, the results are shown in Fig. 3b. Absorbance at $652 \mathrm{~nm}$ increases along with reaction time, and linear relations with $R^{2}$ coefficient close to 1 are obtained by linear regression analysis during the first minute. It is clear that IIM-Fe-SASC has the best peroxidase-like catalytic performance. Then, the catalytic activities expressed in units (U) of IIM-Fe-SASC, NIM-Fe-SASC and NIM were further evaluated (Fig. 3c). The peroxidase-mimic activity of IIMFe-SASC is calculated to be $48.5 \mathrm{U} \mathrm{mg}^{-1}$, which is much higher than that of NIM-Fe-SASC $\left(16.6 \mathrm{U} \mathrm{mg}^{-1}\right)$ and NIM $\left(4.4 \mathrm{U} \mathrm{mg}^{-1}\right)$ and also superior to most of the reported peroxidase-mimic nanomaterials (Table S1). Herein, the added $\mathrm{Fe}$ amount in the precursor was also optimized. As shown in Table S2, the obtained IIM-Fe-SASC by adding $10 \mathrm{mg} \mathrm{Fe}$ precursor own the best enzyme-like activity. As the Fe precursor increases from 5 to $10 \mathrm{mg}$, the single-atom iron will also be increased, boosting the enzyme-like activity. However, adding an excessive amount of Fe precursor exceeds the maximum confinement capability of $\mathrm{SiO}_{\mathrm{x}}$ matrix, resulting in forming some iron crystal during pyrolysis process (Fig. S6). The peroxidase-mimic activity of IIM-Fe-SASC is more than tenfold than that of NIM, which further illustrates that the activity is derived from $\mathrm{Fe}-\mathrm{N}_{x}$ active sites. What's more, the huge activity gap between IIM-Fe-SASC and NIM-Fe-SASC proves that applying IIT results in relatively high-density atomic $\mathrm{Fe}-\mathrm{N}_{\mathrm{x}}$ active sites, thus boosting the peroxidase-like performance. For comparison, the specific activity of natural HRP is evaluated to be $263.8 \mathrm{mg} \mathrm{mL}^{-1}$ under the same process, which is in accordance with the manufacture's value $\left(\geq 250 \mathrm{U} \mathrm{mg}^{-1}\right)$, and the specific activity of the IIM-Fe-SASC is approaching that of natural HRP.

By comparing the detection performances of $\mathrm{H}_{2} \mathrm{O}_{2}$ $(100 \mathrm{mM})$ and its interfering substrates $(500 \mathrm{mM})$ of cysteine, ascorbic acid, $\mathrm{NaCl}$, glucose, alanine and sucrose, the selectivity of IIM-Fe-SASC toward $\mathrm{H}_{2} \mathrm{O}_{2}$ was revealed and shown in Fig. 3d, indicating IIM-Fe-SASC has a satisfactory selectivity toward $\mathrm{H}_{2} \mathrm{O}_{2}$. Furthermore, the steady-state kinetics curves of IIM-Fe-SASC toward $\mathrm{H}_{2} \mathrm{O}_{2}$ and TMB substrates were obtained and shown in 
(a)
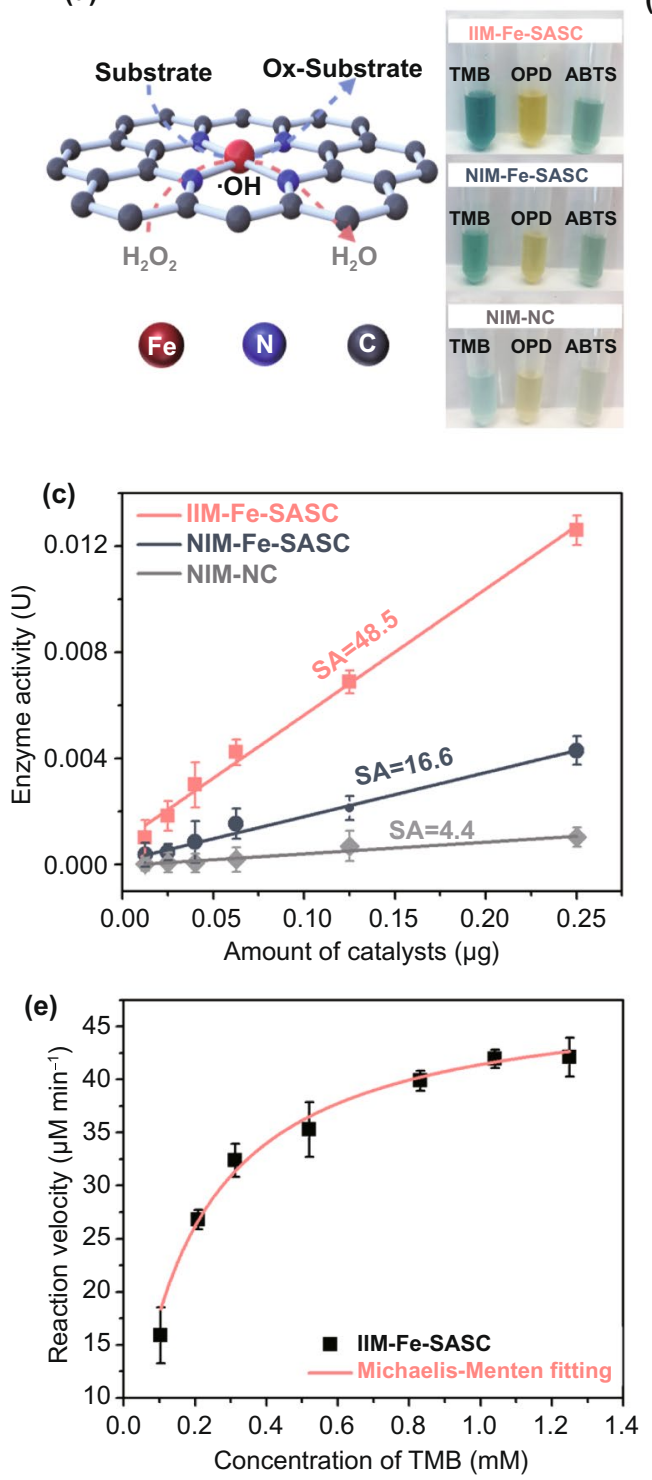
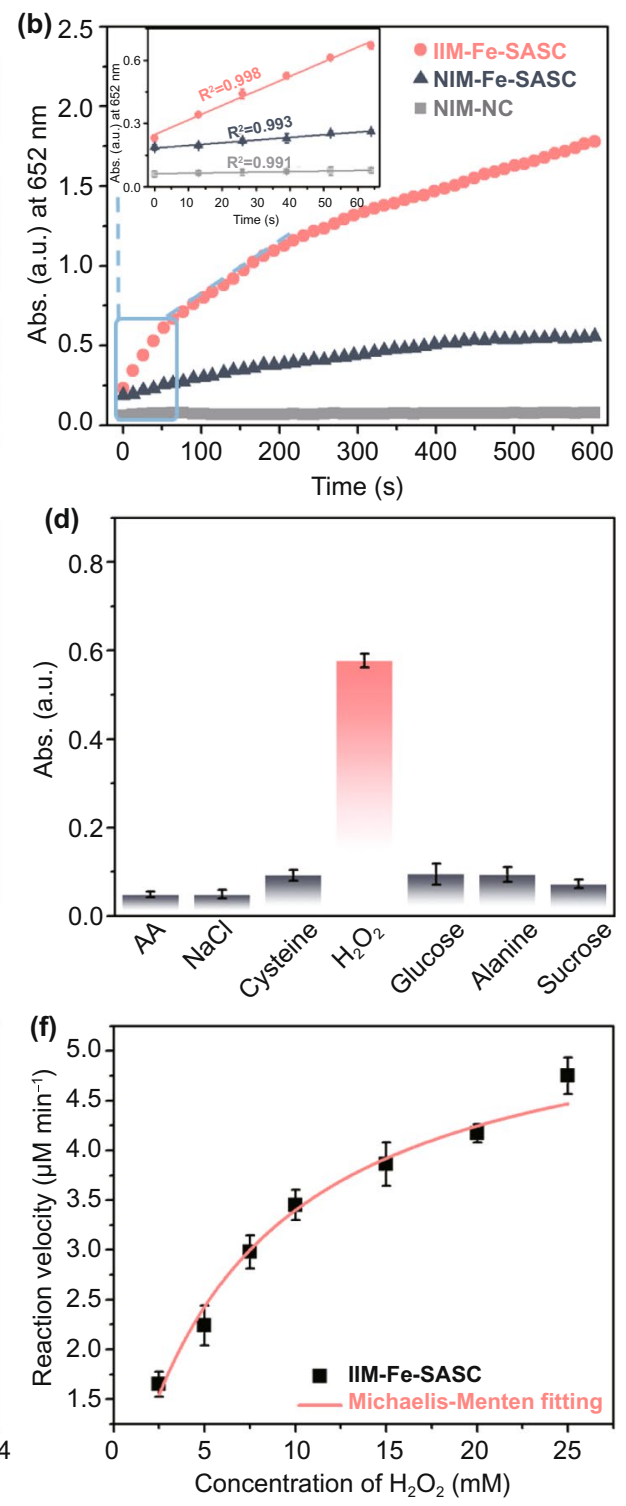

Fig. 3 a Mechanism illustration of IIM-Fe-SASC catalyzed and chromogenic reaction with different substrates. b Absorbance-time curves and magnified initial linear portion of TMB chromogenic reaction catalyzed by IIM-Fe-SASC, NIM-Fe-SASC, and NIM. c Specific activities of IIM-Fe-SASC, NIM-Fe-SASC, and NIM. d Specificity evaluation of IIM-Fe-SASC for various interferences. e-f Steady-state kinetics curves of IIM-Fe-SASC toward TMB and $\mathrm{H}_{2} \mathrm{O}_{2}$

Fig. 3e, f, while HRP was used to serve as a reference for comparison (Fig. S7). Typical Michaelis-Menten curves and the double reciprocal plots of initial reaction rates (Fig. S8) are observed. By fitting in Michaelis-Menten model, Michaelis-Menten parameters of IIM-Fe-SASC and nature HRP toward TMB and $\mathrm{H}_{2} \mathrm{O}_{2}$ are obtained (Table S3). IIM-Fe-SASC shows a comparable $K_{\mathrm{m}}$ toward $\mathrm{H}_{2} \mathrm{O}_{2}$ compared with natural $\mathrm{HRP}$, and $K_{\mathrm{m}}$ of
IIM-Fe-SASC to TMB is lower than that of natural HRP, indicating the IIM-Fe-SASC has a higher affinity toward TMB and a similar affinity level toward $\mathrm{H}_{2} \mathrm{O}_{2}$. We further analyzed the potential effects of harsh environments of temperature and $\mathrm{pH}$ on the peroxidase-like activity of IIM-Fe-SASC. As shown in Fig. S9, IIM-Fe-SASC can preserve their activity in a wide $\mathrm{pH}$ range of $2.5-8.5$, while maintained above $80 \%$ activity from 4 to $80{ }^{\circ} \mathrm{C}$, 
which shows satisfaction robustness against the harsh environment.

\subsection{Mechanisms for Peroxidase-like Activity}

Thiocyanate ions $\left(\mathrm{SCN}^{-}\right)$were used to evaluate the role of single-atom $\mathrm{Fe}$ in catalytic efficiency because $\mathrm{SCN}^{-}$and Fe-centered catalytic sites can form a stable chelate complex, thereby block Fe activity sites and fail to decompose $\mathrm{H}_{2} \mathrm{O}_{2}$. The mechanism illustration is shown in Fig. 4a [46]. As shown in Fig. 4b, the inhibitory effect of peroxidase-like activity is significantly enhanced with the increase in $\mathrm{SCN}^{-}$. These results further prove that the peroxidase-like activity of Fe-SASC is mainly generated from the atomically dispersed $\mathrm{Fe}-\mathrm{N}_{x}$ active sites, which is consistent with the huge specific activity difference in Fig. 3c. The active intermediates were also investigated using various scavengers (Fig. 4c-f). In Fig. 4c, the absorbance value of ox-TMB decreases significantly with the addition of $\mathrm{NaN}_{3}$, indicating that the participation of $\bullet \mathrm{OH} /{ }^{1} \mathrm{O}_{2}$ is related to the oxidation coloration reaction
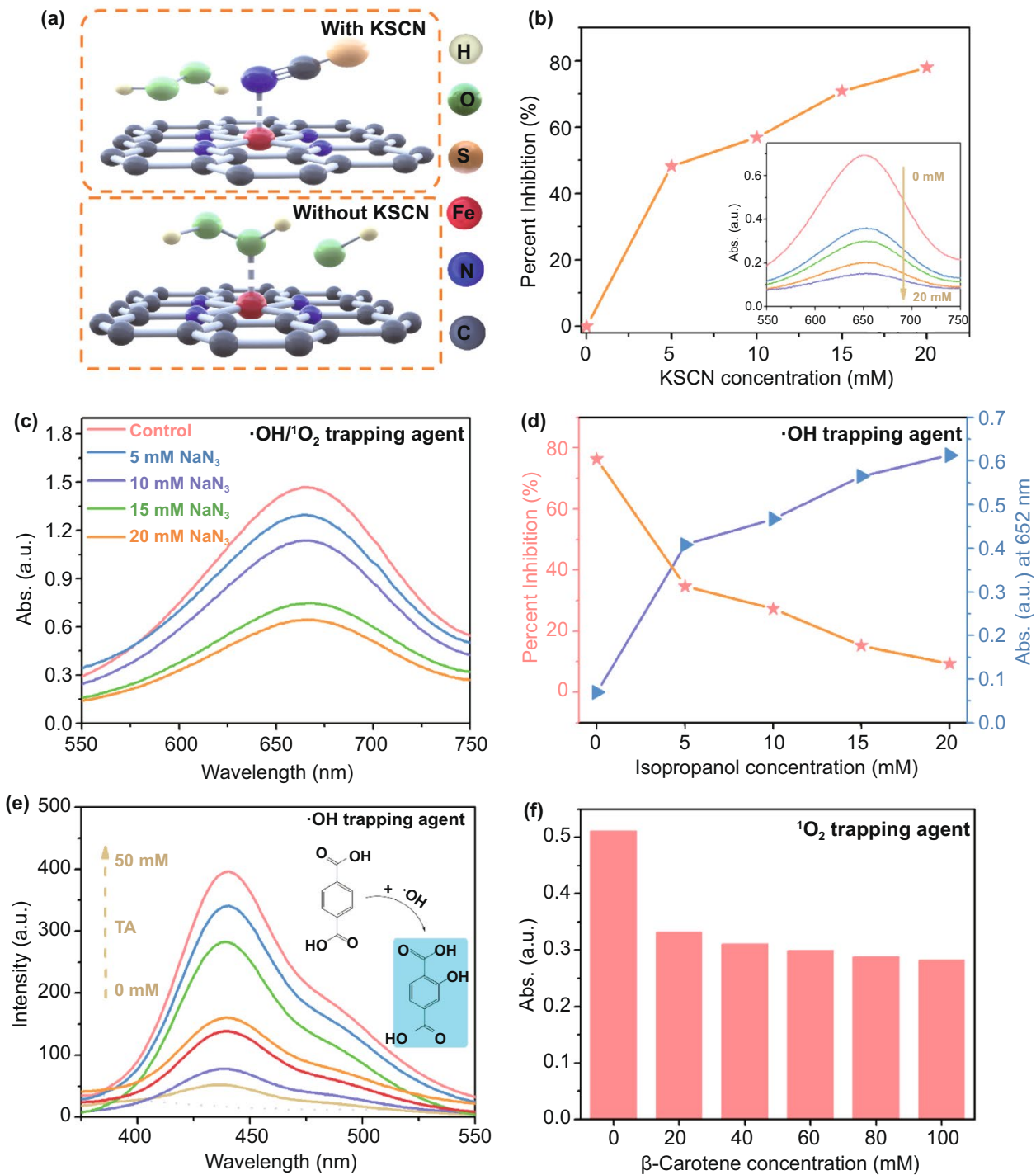

Fig. 4 a Schematic illustration of the mechanism of KSCN influence. b Percent inhibition and absorption change of IIM-Fe$\mathrm{SASC}+\mathrm{TMB}+\mathrm{H}_{2} \mathrm{O}_{2}$ solution upon the addition of various concentrations of KSCN. c Absorbance changes after adding various amounts of $\mathrm{NaN}_{3}$. d Percent inhibition and absorption change after adding isopropanol. e TA as a $\bullet \mathrm{OH}$ fluorescent probe. $\mathbf{f}$ Absorption change after adding $\beta$-carotene with different concentrations 
[47]. The generated $\bullet \mathrm{OH}$ was detected by the enhanced isopropanol inhibition ability (Fig. 4d). The higher fluorescent signal of terephthalic acid (TA) catalyzed by IIMFe-SASC nanoprobe demonstrated that more $\bullet \mathrm{OH}$ is generated (Fig. 4e) [48, 49]. Besides, experimental results related to $\beta$-carotene verified the little presence of ${ }^{1} \mathrm{O}_{2}$ (Fig. 4f) [50].

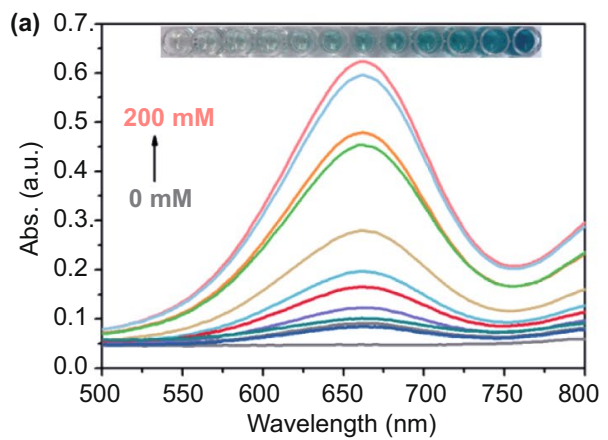

(c)

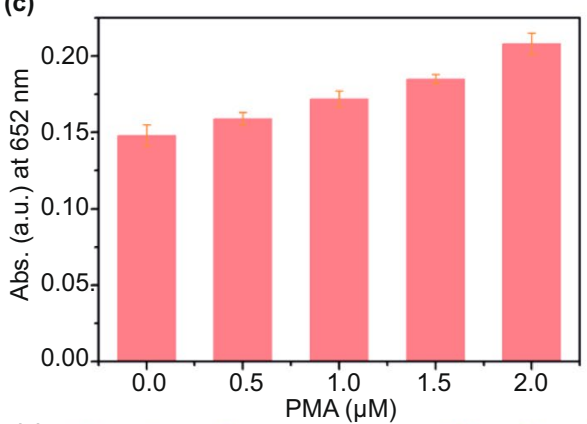

(e)

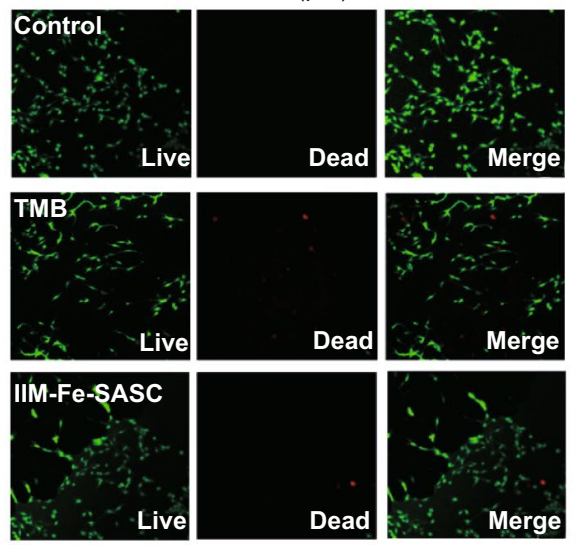

\subsection{Hydrogen Peroxide Detection in Living Cells}

The linear detection range of IIM-Fe-SASC nanoprobe to $\mathrm{H}_{2} \mathrm{O}_{2}$ is determined and the results are shown in Fig. 5a. Accordingly, a fine linear relationship of $\mathrm{H}_{2} \mathrm{O}_{2}$ concentration to absorbance curve is achieved in the range of $0.25-5 \mathrm{mM}$ (Fig. 5b). The MDA-MB-231 breast cancer cells were used for intracellular hydrogen peroxide detection. First, a standard MTT assay was carried out to verify the potential toxicity
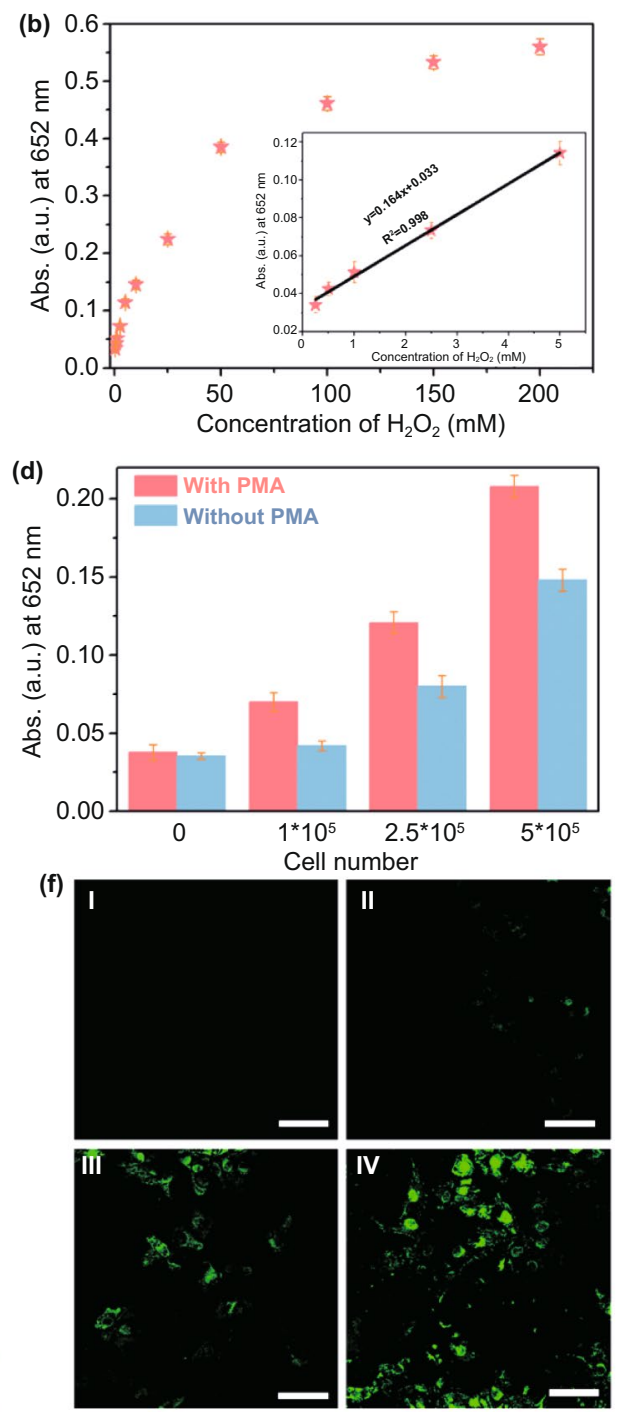

Fig. 5 a UV-vis spectra of TMB oxidized by the IIM-Fe-SASC under different $\mathrm{H}_{2} \mathrm{O}_{2}$ concentrations. b Absorbance changes and linear calibration plot (inset) for $\mathrm{H}_{2} \mathrm{O}_{2}$ detection. c Absorbance values of $\mathrm{H}_{2} \mathrm{O}_{2}$ generated from MDA-MB-231 cells after treating with various concentrations of PMA. d Absorbance comparison toward MDA and PMA-treated MDA-MB-231 cells. e Fluorescence imaging of MDA-MB-231 cancer cells cultured with TMB and IIM-Fe-SASC nanoprobe. f CLSM images of active intermediates generated in MDA-MB-231 cells treated with different amount of IIM-Fe-SASC nanoprobe (I: $0 \mu \mathrm{g} \mathrm{mL}^{-1}$; II: $2.5 \mu \mathrm{g} \mathrm{mL}^{-1}$; III: $5 \mu \mathrm{g} \mathrm{mL}^{-1}$; IV: $10 \mu \mathrm{g} \mathrm{mL}^{-1}$ ). The intracellular ROS generation was detected by the DCFH-DA (Scale bar: $60 \mu \mathrm{m}$ ) 
of TMB and IIM-Fe-SASC (Fig. S10). It is clear that the added TMB has little effect on cell viability. Furthermore, after $24 \mathrm{~h}$ of incubation in IIM-Fe-SASC with a concentration range of $1.0-10 \mu \mathrm{g} \mathrm{mL}^{-1}$, MDA-MB-231 cells can still retain their high viability, revealing the excellent biocompatibility of IIM-Fe-SASC. Adenosine-5-diphosphate (ADP), $N$-formylmethionyl-leucyl-phenylalanine (fMLP) and phorbol-12-myristate-13-acetate (PMA) were used to stimulate MDA-MB-231 cells, and the released $\mathrm{H}_{2} \mathrm{O}_{2}$ was detected (Fig. S11), in which PMA exhibited the optimal stimuli [51]. Then, PMA was then selected to treat MDAMB-231 cells under different concentrations. The results in Fig. 5c show that the absorbance value is highly dependent on PMA concentrations. Furthermore, different cell numbers were treated with or without PMA. A higher colorimetric response is observed as the cell number increased, which can be ascribed to more $\mathrm{H}_{2} \mathrm{O}_{2}$ produced during PMA stimulation (Fig. 5d). Also, according to the $\mathrm{H}_{2} \mathrm{O}_{2}$ detection calibration curve in Fig. $5 \mathrm{~b}, \mathrm{H}_{2} \mathrm{O}_{2}$ concentration produced from the MDA-MB-231 cells $\left(2.5 \times 10^{5}\right.$ cells/plate $)$ is calculated to be $0.535 \mathrm{mM}$, and the average molecule number of $\mathrm{H}_{2} \mathrm{O}_{2}$ released in one cell $\left(N_{0}\right)$ is $3.48 \times 10^{11}$ (calculated by the Avogadro equation: $n=N_{0} / N_{\mathrm{A}}$, in which Avogadro's constant $N_{\mathrm{A}}$ is $6.02 \times 10^{23} \mathrm{~mol}^{-1}$ ). This value is in good agreement with the previous reports $[49,52]$, indicating that the colorimetric detection method based on IIM-Fe-SASC nanoprobe can be used in practical clinic applications.

A standard staining method was also used to evaluate the biocompatibility by a live/dead viability kit. The calcein-AM can combine with the living cell through the cell membrane and green fluorescence can be observed in the cytoplasm through a fluorescent microscope. As shown in Fig. 5e, MDA-MB-231 cells were cultured with IIM-FeSASC nanoprobe and TMB under testing concentrations. The CLSM images show that the TMB has minor effects on cell viability. And for IIM-Fe-SASC nanoprobe, no significant cell viability changes. Intracellular $\mathrm{H}_{2} \mathrm{O}_{2}$ detection was also performed through transporting IIM-Fe-SASC nanoprobe into MDA-MB-231 cells by endocytosis. Since we have already proved that the $\bullet \mathrm{OH}$ and ${ }^{1} \mathrm{O}_{2}$ are active intermediates during the peroxidase-like catalytic reaction, these intracellular reactive oxygen species can be evaluated using a fluorescence probe $2^{\prime}, 7^{\prime}$-dichlorofluorescein diacetate (DCFH-DA) [53]. As illustrated in Fig. 5f, MDA-MB-231 cells show insignificant green fluorescence when incubating with IIM-Fe-SASC nanoprobe. In contrast, obvious green fluorescence is observed in control cells (Fig. 5f-I), suggesting IIM-Fe-SASC nanoprobe can produce massive intracellular active intermediates. In addition, the intensity of the green fluorescence signal is also enhanced with the increasing concentration of nanoprobes. These results further demonstrate the excellent intracellular $\mathrm{H}_{2} \mathrm{O}_{2}$ detection ability of IIM-Fe-SASC nanoprobe.

\section{Conclusion}

In summary, we have used a facile ion-imprinting approach to synthesize a Fe-based single-atom nanoprobe for hydrogen peroxide detection in living cells. The resultant IIM-FeSASC shows better peroxidase-like activity than that of nonimprinted references, demonstrating that the ion-imprinting process can precisely control ion at the atomic level and form numerous well-defined single-atom iron. High sensitivity and specificity of IIM-Fe-SASC nanoprobe have been achieved for colorimetric detection of $\mathrm{H}_{2} \mathrm{O}_{2}$. Furthermore, in situ detection of $\mathrm{H}_{2} \mathrm{O}_{2}$ generated from the MDA-MB-231 cells was performed, exhibiting satisfactory sensitivity and specificity. This work opens a novel and easy route in designing advanced single-atom nanoprobe and expands their biosensing applications.

Acknowledgements This work was supported by a WSU startup fund. XAS measurements were done at beamline 12-BM of the Advanced Photon Source (APS), which is a User Facility operated for the U.S. Department of Energy Office of Science by Argonne National Laboratory under Contract DE-AC02-06CH11357. The authors would like to acknowledge Dr. Suiqiong Li for helpful discussion and editing of the manuscript. We would like to thank the WSU Franceschi Microscopy \& Imaging Center for TEM and SEM measurements.

Open Access This article is licensed under a Creative Commons Attribution 4.0 International License, which permits use, sharing, adaptation, distribution and reproduction in any medium or format, as long as you give appropriate credit to the original author(s) and the source, provide a link to the Creative Commons licence, and indicate if changes were made. The images or other third party material in this article are included in the article's Creative Commons licence, unless indicated otherwise in a credit line to the material. If material is not included in the article's Creative Commons licence and your intended use is not permitted by statutory regulation or exceeds the permitted use, you will need to obtain permission directly from the copyright holder. To view a copy of this licence, visit http://creativecommons.org/licenses/by/4.0/. 
Supplementary Information The online version contains supplementary material available at https://doi.org/10.1007/ s40820-021-00661-z.

\section{References}

1. H. Ohshima, M. Tatemichi, T. Sawa, Chemical basis of inflammation-induced carcinogenesis. Arch. Biochem. Biophys. 417(1), 3-11 (2003). https://doi.org/10.1016/S0003-9861(03) 00283-2

2. J. Bai, X. Jiang, A facile one-pot synthesis of copper sulfidedecorated reduced graphene oxide composites for enhanced detecting of $\mathrm{H}_{2} \mathrm{O}_{2}$ in biological environments. Anal. Chem. 85(17), 8095-8101 (2013). https://doi.org/10.1021/ac400 $659 \mathrm{u}$

3. Q. Chen, C. Liang, X. Sun, J. Chen, Z. Yang et al., $\mathrm{H}_{2} \mathrm{O}_{2}$-responsive liposomal nanoprobe for photoacoustic inflammation imaging and tumor theranostics via in vivo chromogenic assay. Proc. Natl. Acad. Sci. USA 114(21), 5343-5348 (2017). https://doi.org/10.1073/pnas.1701976114

4. T. Finkel, M. Serrano, M.A. Blasco, The common biology of cancer and ageing. Nature 448(7155), 767-774 (2007). https:// doi.org/10.1038/nature05985

5. T. Zhang, Y. Xing, Y. Song, Y. Gu, X. Yan et al., Aupt/mofgraphene: a synergistic catalyst with surprisingly high peroxidase-like activity and its application for $\mathrm{H}_{2} \mathrm{O}_{2}$ detection. Anal. Chem. 91(16), 10589-10595 (2019). https://doi.org/10.1021/ acs.analchem.9b01715

6. J. Liu, J. Liang, C. Wu, Y. Zhao, A doubly-quenched fluorescent probe for low-background detection of mitochondrial $\mathrm{H}_{2} \mathrm{O}_{2}$. Anal. Chem. 91(10), 6902-6909 (2019). https://doi.org/ 10.1021/acs.analchem.9b01294

7. Z. Deng, Y. Qian, Y. Yu, G. Liu, J. Hu et al., Engineering intracellular delivery nanocarriers and nanoreactors from oxidation-responsive polymersomes via synchronized bilayer cross-linking and permeabilizing inside live cells. J. Am. Chem. Soc. 138(33), 10452-10466 (2016). https://doi.org/ 10.1021/jacs.6b04115

8. Q. Fu, H. Saltsburg, M. Flytzani-Stephanopoulos, Active nonmetallic au and pt species on ceria-based water-gas shift catalysts. Science 301(5635), 935-938 (2003). https://doi.org/ 10.1126/science. 1085721

9. C. Zhu, S. Fu, Q. Shi, D. Du, Y. Lin, Single-atom electrocatalysts. Angew. Chem. Int. Ed. 56(45), 13944-13960 (2017). https://doi.org/10.1002/anie.201703864

10. X. Wei, X. Luo, N. Wu, W. Gu, Y. Lin et al., Recent a dvances in synergistically enhanced single-atomic site catalysts for boosted oxygen reduction reaction. Nano Energy 84, 105817 (2021). https://doi.org/10.1016/j.nanoen.2021.105817

11. B. Qiao, A. Wang, X. Yang, L.F. Allard, Z. Jiang et al., Singleatom catalysis of co oxidation using $\mathrm{Pt}_{1} / \mathrm{FeO}_{\mathrm{x}}$. Nat. Chem. 3(8), 634-641 (2011). https://doi.org/10.1038/nchem.1095
12. S. Ji, Y. Chen, X. Wang, Z. Zhang, D. Wang et al., Chemical synthesis of single atomic site catalysts. Chem. Rev. 120(21), 11900-11955 (2020). https://doi.org/10.1021/acs.chemrev. $9 \mathrm{~b} 00818$

13. F.X. Hu, T. Hu, S. Chen, D. Wang, Q. Rao et al., Singleatom cobalt-based electrochemical biomimetic uric acid sensor with wide linear range and ultralow detection limit. Nano-Micro Lett. 13(1), 7 (2020). https://doi.org/10.1007/ s40820-020-00536-9

14. H. Zhang, W. Zhou, T. Chen, B.Y. Guan, Z. Li et al., A modular strategy for decorating isolated cobalt atoms into multichannel carbon matrix for electrocatalytic oxygen reduction. Energy Environ. Sci. 11(8), 1980-1984 (2018). https://doi.org/ 10.1039/C8EE00901E

15. L. Huang, J. Chen, L. Gan, J. Wang, S. Dong, Single-atom nanozymes. Sci. Adv. 5(5), 5490 (2019). https://doi.org/10. 1126/sciadv.aav5490

16. L. Jiao, H. Yan, Y. Wu, W. Gu, C. Zhu et al., When nanozymes meet single-atom catalysis. Angew. Chem. Int. Ed. 132(7), 2585-2596 (2020). https://doi.org/10.1002/ange.201905645

17. L. Jiao, W. Xu, Y. Wu, H. Yan, W. Gu et al., Single-atom catalysts boost signal amplification for biosensing. Chem. Soc. Rev. 50(2), 750-765 (2021). https://doi.org/10.1039/D0CS0 $0367 \mathrm{~K}$

18. W. Wu, L. Huang, E. Wang, S. Dong, Atomic engineering of single-atom nanozymes for enzyme-like catalysis. Chem. Sci. 11(36), 9741-9756 (2020). https://doi.org/10.1039/D0SC0 $3522 \mathrm{~J}$

19. X. Niu, Q. Shi, W. Zhu, D. Liu, H. Tian et al., Unprecedented peroxidase-mimicking activity of single-atom nanozyme with atomically dispersed $\mathrm{Fe}-\mathrm{N}_{\mathrm{x}}$ moieties hosted by mof derived porous carbon. Biosens. Bioelectron. 142, 111495 (2019). https://doi.org/10.1016/j.bios.2019.111495

20. Y. Chen, L. Jiao, H. Yan, W. Xu, Y. Wu et al., Hierarchically porous s/n codoped carbon nanozymes with enhanced peroxidase-like activity for total antioxidant capacity biosensing. Anal. Chem. 92(19), 13518-13524 (2020). https://doi.org/10. 1021/acs.analchem.0c02982

21. L. Jiao, W. Xu, Y. Zhang, Y. Wu, W. Gu et al., Boron-doped $\mathrm{Fe}-\mathrm{N}-\mathrm{C}$ single-atom nanozymes specifically boost peroxidaselike activity. Nano Today 35, 100971 (2020). https://doi.org/ 10.1016/j.nantod.2020.100971

22. N. Cheng, J.-C. Li, D. Liu, Y. Lin, D. Du, Single-atom nanozyme based on nanoengineered $\mathrm{Fe}-\mathrm{N}-\mathrm{C}$ catalyst with superior peroxidase-like activity for ultrasensitive bioassays. Small 15(48), 1901485 (2019). https://doi.org/10.1002/smll. 201901485

23. Z. Lyu, S. Ding, N. Zhang, Y. Zhou, N. Cheng et al., Singleatom nanozymes linked immunosorbent assay for sensitive detection of A $\beta 1-40$ : A biomarker of alzheimer's disease. Research 2020, 4724505 (2020). https://doi.org/10.34133/ 2020/4724505

24. R. Jiang, L. Li, T. Sheng, G. Hu, Y. Chen et al., Edge-site engineering of atomically dispersed $\mathrm{Fe}-\mathrm{N}_{4}$ by selective $\mathrm{C}-\mathrm{N}$ bond cleavage for enhanced oxygen reduction reaction activities. J. 
Am. Chem. Soc. 140(37), 11594-11598 (2018). https://doi. org/10.1021/jacs.8b07294

25. H. Zhang, J. Li, S. Xi, Y. Du, X. Hai et al., A graphenesupported single-atom fen5 catalytic site for efficient electrochemical $\mathrm{CO}_{2}$ reduction. Angew. Chem. Int. Ed. 131(42), 15013-15018 (2019). https://doi.org/10.1002/ange.201906079

26. J. Han, J. Bian, C. Sun, Recent advances in single-atom electrocatalysts for oxygen reduction reaction. Research (2020). https://doi.org/10.34133/2020/9512763

27. Y. Qu, L. Wang, Z. Li, P. Li, Q. Zhang et al., Ambient synthesis of single-atom catalysts from bulk metal via trapping of atoms by surface dangling bonds. Adv. Mater. 31(44), 1904496 (2019). https://doi.org/10.1002/adma.201904496

28. J.-C. Li, Z.-Q. Yang, D.-M. Tang, L. Zhang, P.-X. Hou et al., $\mathrm{N}$-doped carbon nanotubes containing a high concentration of single iron atoms for efficient oxygen reduction. NPG Asia Mater. 10(1), e461 (2018). https://doi.org/10.1038/am.2017. 212

29. J. Han, H. Bao, J.-Q. Wang, L. Zheng, S. Sun et al., 3d $\mathrm{N}$-doped ordered mesoporous carbon supported single-atom Fe-N-C catalysts with superior performance for oxygen reduction reaction and zinc-air battery. Appl. Catal. B Environ. 280, 119411 (2021). https://doi.org/10.1016/j.apcatb.2020.119411

30. X.-F. Yang, A. Wang, B. Qiao, J. Li, J. Liu et al., Singleatom catalysts: a new frontier in heterogeneous catalysis. Acc. Chem. Res. 46(8), 1740-1748 (2013). https://doi.org/10.1021/ ar300361m

31. J. Fu, L. Chen, J. Li, Z. Zhang, Current status and challenges of ion imprinting. J. Mater. Chem. A 3(26), 13598-13627 (2015). https://doi.org/10.1039/C5TA02421H

32. N. Zhang, N. Zhang, Y. Xu, Z. Li, C. Yan et al., Molecularly imprinted materials for selective biological recognition. Macromol. Rapid Commun. 40(17), 1900096 (2019). https://doi. org/10.1002/marc.201900096

33. S. Ding, Z. Lyu, X. Niu, Y. Zhou, D. Liu et al., Integrating ionic liquids with molecular imprinting technology for biorecognition and biosensing: a review. Biosens. Bioelectron. 149, 111830 (2020). https://doi.org/10.1016/j.bios.2019. 111830

34. Y. Berro, S. Gueddida, Y. Bouizi, C. Bellouard, E.-E. Bendeif et al., Imprinting isolated single iron atoms onto mesoporous silica by templating with metallosurfactants. J. Colloid Interface Sci. 573, 193-203 (2020). https://doi.org/10.1016/j.jcis. 2020.03.095

35. S. Ding, Z. Lyu, H. Zhong, D. Liu, E. Sarnello et al., An ionimprinting derived strategy to synthesize single-atom iron electrocatalysts for oxygen reduction. Small (2020). https:// doi.org/10.1002/smll.202004454

36. H. Zhang, W. Zhou, X.F. Lu, T. Chen, X.W. Lou, Implanting isolated $\mathrm{Ru}$ atoms into edge-rich carbon matrix for efficient electrocatalytic hydrogen evolution. Adv. Energy Mater. 10(23), 2000882 (2020). https://doi.org/10.1002/aenm.20200 0882

37. J. Li, H. Zhang, W. Samarakoon, W. Shan, D.A. Cullen et al., Thermally driven structure and performance evolution of atomically dispersed $\mathrm{FeN}_{4}$ sites for oxygen reduction. Angew.
Chem. Int. Ed. 131(52), 19147-19156 (2019). https://doi.org/ 10.1002/ange. 201909312

38. R. Li, Z. Wei, X. Gou, Nitrogen and phosphorus dual-doped graphene/carbon nanosheets as bifunctional electrocatalysts for oxygen reduction and evolution. ACS Catal. 5(7), 41334142 (2015). https://doi.org/10.1021/acscatal.5b00601

39. S. Yi, X. Qin, C. Liang, J. Li, R. Rajagopalan et al., Insights into kmno4 etched n-rich carbon nanotubes as advanced electrocatalysts for zn-air batteries. Appl. Catal. B Environ. 264, 118537 (2020). https://doi.org/10.1016/j.apcatb.2019.118537

40. J. Han, X. Meng, L. Lu, J. Bian, Z. Li et al., Single-atom $\mathrm{Fe}-\mathrm{N}_{\mathrm{x}}-\mathrm{C}$ as an efficient electrocatalyst for zinc-air batteries. Adv. Funct. Mater. 29(41), 1808872 (2019). https://doi.org/ 10.1002/adfm.201808872

41. L. Tao, Q. Wang, S. Dou, Z. Ma, J. Huo et al., Edge-rich and dopant-free graphene as a highly efficient metal-free electrocatalyst for the oxygen reduction reaction. Chem. Commun. 52(13), 2764-2767 (2016). https://doi.org/10.1039/C5CC0 9173J

42. P. Hao, Z. Zhao, Y. Leng, J. Tian, Y. Sang et al., Graphenebased nitrogen self-doped hierarchical porous carbon aerogels derived from chitosan for high performance supercapacitors. Nano Energy 15, 9-23 (2015). https://doi.org/10.1016/j. nanoen.2015.02.035

43. J. Wang, F. Ciucci, Boosting bifunctional oxygen electrolysis for $\mathrm{N}$-doped carbon via bimetal addition. Small 13(16), 1604103 (2017). https://doi.org/10.1002/smll.201604103

44. H. Zhang, Y. Liu, T. Chen, J. Zhang, J. Zhang et al., Unveiling the activity origin of electrocatalytic oxygen evolution over isolated $\mathrm{Ni}$ atoms supported on a N-doped carbon matrix. Adv. Mater. 31(48), 1904548 (2019). https://doi.org/10.1002/adma. 201904548

45. N. Leonard, W. Ju, I. Sinev, J. Steinberg, F. Luo et al., The chemical identity, state and structure of catalytically active centers during the electrochemical $\mathrm{CO}_{2}$ reduction on porous Fe-nitrogen-carbon (Fe-N-C) materials. Chem. Sci. 9(22), 5064-5073 (2018). https://doi.org/10.1039/C8SC00491A

46. Y. Wang, K. Qi, S. Yu, G. Jia, Z. Cheng et al., Revealing the intrinsic peroxidase-like catalytic mechanism of heterogeneous single-atom Co-MoS 2 . Nano-Micro Lett. 11(1), 102 (2019). https://doi.org/10.1007/s40820-019-0324-7

47. Y. Zhan, Y. Zeng, L. Li, L. Guo, F. Luo et al., $\mathrm{Cu}^{2+}$-modified boron nitride nanosheets-supported subnanometer gold nanoparticles: an oxidase-mimicking nanoenzyme with unexpected oxidation properties. Anal. Chem. 92(1), 1236-1244 (2020). https://doi.org/10.1021/acs.analchem.9b04384

48. H. Wang, S. Jiang, W. Shao, X. Zhang, S. Chen et al., Optically switchable photocatalysis in ultrathin black phosphorus nanosheets. J. Am. Chem. Soc. 140(9), 3474-3480 (2018). https://doi.org/10.1021/jacs.8b00719

49. L. Jiao, J. Wu, H. Zhong, Y. Zhang, W. Xu et al., Densely isolated $\mathrm{FeN}_{4}$ sites for peroxidase mimicking. ACS Catal. 10(11), 6422-6429 (2020). https://doi.org/10.1021/acscatal.0c01647

50. H. Tamura, H. Ishikita, Quenching of singlet oxygen by carotenoids via ultrafast superexchange dynamics. J. Phy. Chem. A 
124(25), 5081-5088 (2020). https://doi.org/10.1021/acs.jpca. 0c02228

51. Q. Shi, Y. Song, C. Zhu, H. Yang, D. Du et al., Mesoporous Pt nanotubes as a novel sensing platform for sensitive detection of intracellular hydrogen peroxide. ACS Appl. Mater. Interfaces 7(43), 24288-24295 (2015). https://doi.org/10.1021/ acsami.5b08146

52. L. Jiao, W. Xu, H. Yan, Y. Wu, C. Liu et al., Fe-N-C single-atom nanozymes for the intracellular hydrogen peroxide detection. Anal. Chem. 91(18), 11994-11999 (2019). https:// doi.org/10.1021/acs.analchem.9b02901

53. X. Cai, S. Ding, Q. Shi, Z. Lyu, D. Liu et al., Eyeball-like yolkshell bimetallic nanoparticles for synergistic photodynamicphotothermal therapy. ACS Appl. Bio Mater. 3(9), 5922-5929 (2020). https://doi.org/10.1021/acsabm.0c00624 